\title{
Fast Generation Algorithm of Digital Hologram based Depth Difference Temporal Filtering
}

\author{
Hyun-Jun Choi \\ Electronic Eng., Mokpo National Maritime University, \\ Mokpo, Jeonnam, 530729, Korea \\ hjchoi@mmu.ac.kr
}

\begin{abstract}
A CGH (Computer-Generated Hologram) is produced by calculating the interference pattern between the object wave and the reference wave. Thus it has been developed to get some ideal characteristics of CGH that are not possible in reality or to test the characteristics of a hologram. To generate a CGH with the hologram resolution of $(M \times N)$ for a $3 D$ object having $K$ light source, basically $(N \times N \times K)$ calculational iterations are necessary. This paper proposes an algorithm that increases the speed of generating a digital hologram using a depth different temporal filtering (DDTF) of a depth video sequence. The proposed algorithm is a technique that performs CGH operation with only calculation the different depth values between the depth video frames. The experimental results showed that the proposed algorithm increased operation speed by $65 \%$ over the technique using the conventional CGH equation.
\end{abstract}

Keywords: Digital hologram, Computer-generated hologram, CGH, Depth-map

\section{Introduction}

The applications of holograms are being extended to a wide range of devices and fields, from stereopsis displays, high-density memory technology, to high-speed parallel computing using light [1-3]. Holography is expected to be a core technology in next-generation displays just as semiconductor technology became the basis of the digital revolution. After it was introduced in the early 1940s, holography has attracted the interest of many researchers because of its ability to record three-dimensional information [4]. However, the existing holography stored and distributed in the form of analogue films has many constraints with respect to it being commercialized, because it uses a laser as the coherent light that requires limited space, such as a darkroom, to record interference patterns, and is very sensitive to the surrounding environment, such as vibrations, thus requiring a stable optical system. These problems have gradually been overcome with the digitization of devices that generate, acquire, store, process, and display holograms. In particular, it is relatively easy to create a hologram by using computer-generated holography $(\mathrm{CGH})$ techniques. $\mathrm{CGH}$ techniques use a ray-tracing method to calculate the diffraction of light. This method considers the object as a set of multiple points and then sums these points after calculating the intermediate hologram for each point. Consequently, this method requires a considerably large amount of 
calculation. If it is intended to generate a large number of digital holograms, the amount of calculation and the time required increase exponentially. Such a problem is a significant obstacle in a real-time hologram service [5-7].

This paper propose a method to reduce the calculation time that would otherwise increase dramatically when a CGH calculation is carried out for multiple pages of depth maps for a real-time hologram service.

The proposed method includes a depth difference temporal filtering (DDTF) process that separates the difference coordinate (DC) and common coordinate (CC) by searching the temporal redundancy between adjacent images in the time domain of the depth-map video, a process of conducting the $\mathrm{CGH}$ calculation for each of the depth-map frames' coordinates corresponding to the separated $\mathrm{DC}$ and $\mathrm{CC}$, and a process of generating the final digital hologram by summing the generated intermediate holograms. The DDTF, which searches redundancy between adjacent depth-map frames, is performed after setting two to five depthmap frames as one group. The group of depth maps is defined as a group of depths (GoD).

This paper proposes four methods of DDTF CGH techniques-from Method 1 to Method 4depending on the size of GoD, and verifies the performance with various depth-map videos. The depth-map videos that were used for verifying the performance of the proposed algorithm were recordings of objects rotating in place and of ones moving in horizontal directions.

This paper is organized as follows. Section 2 introduces the CGH technique, and Section 3 explains the proposed DDTF CGH algorithm. The experiment results of the proposed algorithm are described in Section 4, and based on this, Section 5 presents the conclusion.

$$
I_{\alpha}=\sum_{j}^{N} A_{j} \cos \left(k \sqrt{\left(p x_{\alpha}-p x_{j}\right)^{2}+\left(p y_{\alpha}-p y_{j}\right)^{2}+z_{j}^{2}}\right)
$$

\section{Computer-generated Hologram}

This section describes the previous CGH calculation method and the one using the recursive addition system.

The CGH generating equation is defined as Eq. (1),

where, $\alpha$ or $j$ indicates a particular point on the hologram or 3D object respectively, $k$ is the wave number of the reference wave defined as $2 \pi / \lambda$, p represents the pixel pitch of the hologram, and $(x \alpha, y \alpha)$ and $(x j, y j, z j)$ represent the coordinates of the hologram and $3 \mathrm{D}$ object respectively[5,8].

Figure 1 shows a sample coordinate array system for the $3 \mathrm{D}$ object and the digital hologram to apply the CGH method, where the $3 \mathrm{D}$ object consists of $2 \times 2$, and the digital hologram is captured as $4 \times 4$ in size. To generate a digital hologram with this setup, the calculation of Eq. (1) must be carried out $64(=2 \times 2 \times 4 \times 4)$ times. 


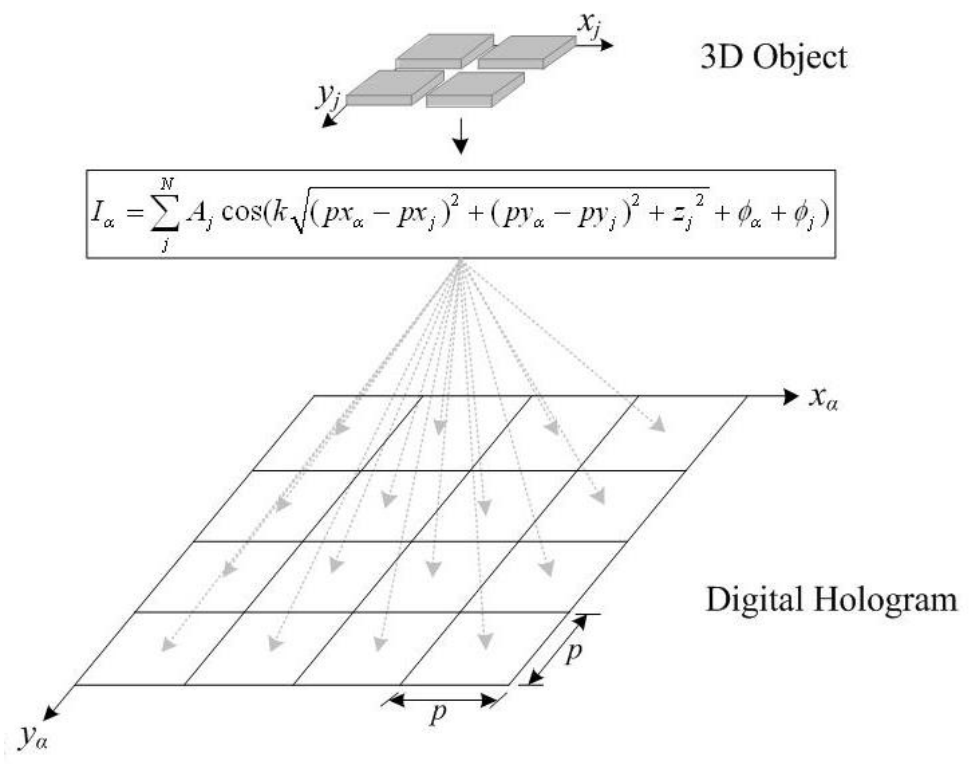

Figure 1. CGH operation of the conventional method

\section{Proposed Algorithm}

As described above, it takes a great deal of time to generate a digital hologram by using the CGH calculation method. While expanding to multiple pages of depth-map frames, one can intuitively predict the increase in the calculation time. To solve these problems, this paper proposes a method to generate a digital hologram video at a high speed by searching the temporal redundancy between the depth-map frames. In this section, the temporal redundancy between the depth-map frames is reviewed, and based on these results; four DDTF CGH algorithms depending on the size of GoD are proposed.

\subsection{Temporal Redundancy between Depth-Map Frames}

A substantial similarity exists between adjacent images in the time domain of a general natural video [9]. In the case of an MPEG-based video compression method, intra-coding is performed on the first image ( $\mathrm{t}$ ) by using the temporal redundancy, and from the second image $(t+1)$, the residual image from the first image is inter-coded and transmitted. If redundancy is found in the time domain in the depth-map video, which is a set of depth-map images, as in a general natural video, this characteristic can be used while generating holograms.

Figure 2 illustrates the experimental procedure for measuring temporal redundancy by configuring two depth maps as one $\mathrm{GoD}$ for the depth-map video consisting of $\mathrm{n}$ depth-map frames. This experiment is carried out to find the $\mathrm{DC}$ and the $\mathrm{CC}$ information by calculating the similarity between adjacent frames through a process of difference generator (DG) for $n$ depth-map frames. 


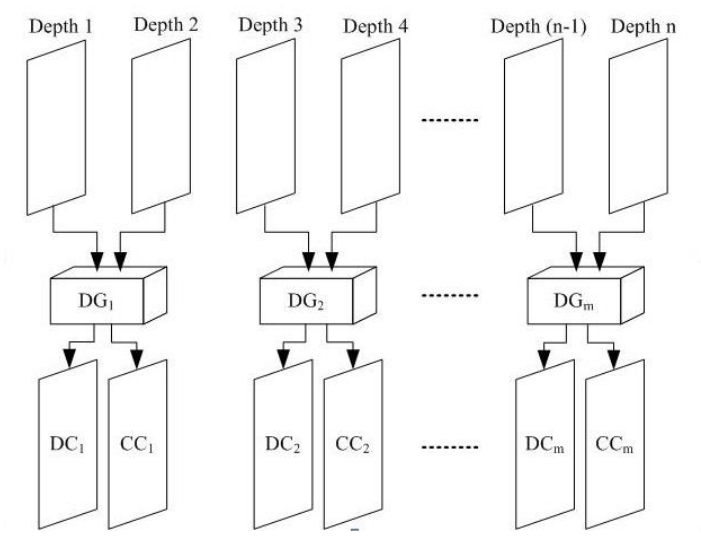

Figure 2. Experimental Procedure for Calculating Temporal Redundancy

Figure 3 shows the experimental image examples used for measuring redundancy in the time domain for the depth-map videos. Figures 3(a) and (b) show the depth-map videos of rotating objects recorded using a depth-map camera, and Figure 3(c) shows a depth-map video of a horizontally moving object. These images exemplarily show partial frames of the depth-map video frames used in the experiment.

Figure 4 shows the result of calculating temporal redundancy in the time domain for the three depth-map videos. Each graph in Figure 4 represents the number of same values between adjacent frames out of the selected ten frames from the entire set of video frames. The $x$-domain in this graph represents the randomly selected video frames, and the $y$-domain represents the number of same depth maps in the adjacent frames. Herein, the size of GoD was set to range between two and five based on the randomly selected ten frames to measure the number of same depth maps within one GoD.
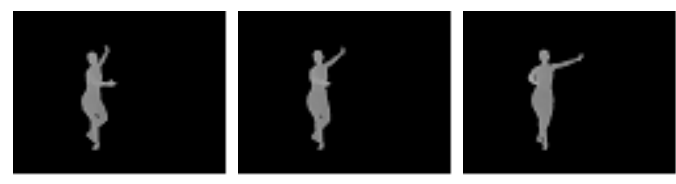

(a)
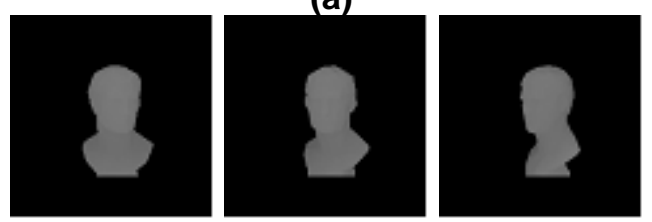

(b)
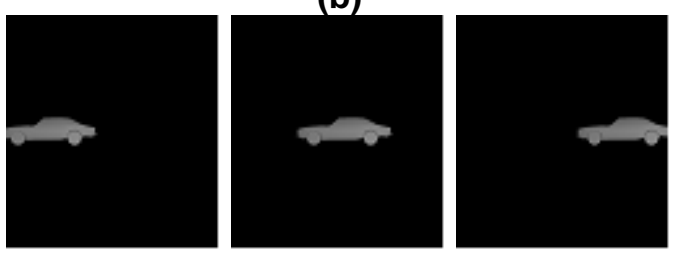

(c)

Figure 3. Experimental Image Examples: (a) Ballet, (b) David, and (c) Camaro 
In the case of Figure 4(a), since the depth-map video used in the experiment consisted only eleven frames, if $\mathrm{GoD}=2$, then 5 frames; $\mathrm{GoD}=3,3$ frames; $\mathrm{GoD}=4,2$ frames; and $\mathrm{GoD}=$ 5, 2 frames were selected for measuring the number of depth maps after the GoD was structured on the basis of these frames. In the case of Figure 4(b), it was confirmed that a considerable number of same values of depth maps existed within the same GoD because an object rotating in the same place was recorded. Figure 4(c) shows the result of the experiment conducted on the depth-map video in which an object moving horizontally is recorded. It was confirmed that the number of same depth maps decreased with an increase in the size of GoD because this depth-map video consisted of 38 frames and the frame rate was relatively low. It was found that the number of same depth maps was the highest when only two frames were used for composing the GoD from among all the experimental data of Figure 4.

\subsection{Depth Different Temporal Filtering CGH (DDTF CGH)}

As discussed in Section 3.1, a substantial similarity existed between the adjacent frames in the depth-map video as in general natural videos. On the basis of these characteristics, we propose an algorithm that speeds up the $\mathrm{CGH}$ calculation for the depth-map videos for a holographic video service. In this paper, the depth-map video sequence was separated into GoDs, and then, the similarities between the depth-map frames were discovered within each GoD by using DDTF.

Figure 5 illustrates four techniques to control the number of depth-map frames ranging from two to five within a GoD depending on the total number of depth-map video frames.

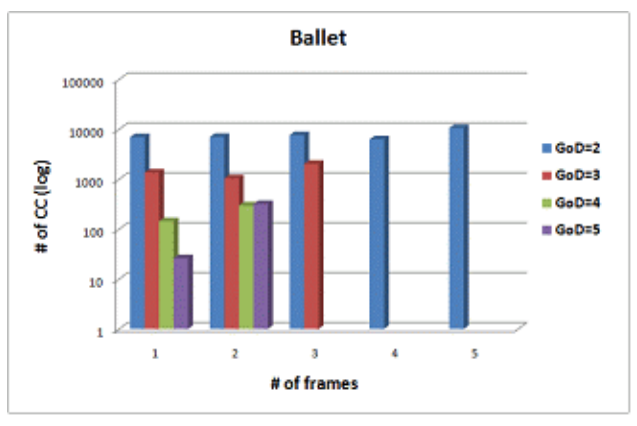

(a)

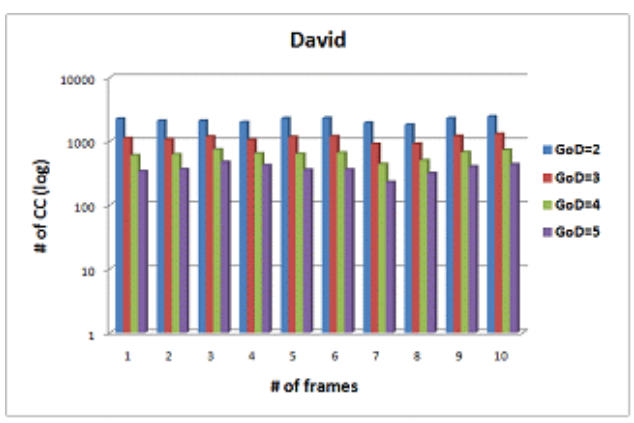

(b)

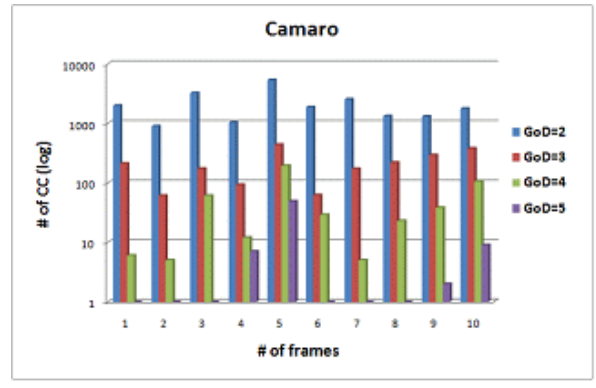

(c)

Figure 4. Redundancy Test Results in the Time Domain of the Depth-Map Video: (a) Ballet, (b) David, and (c) Camaro 


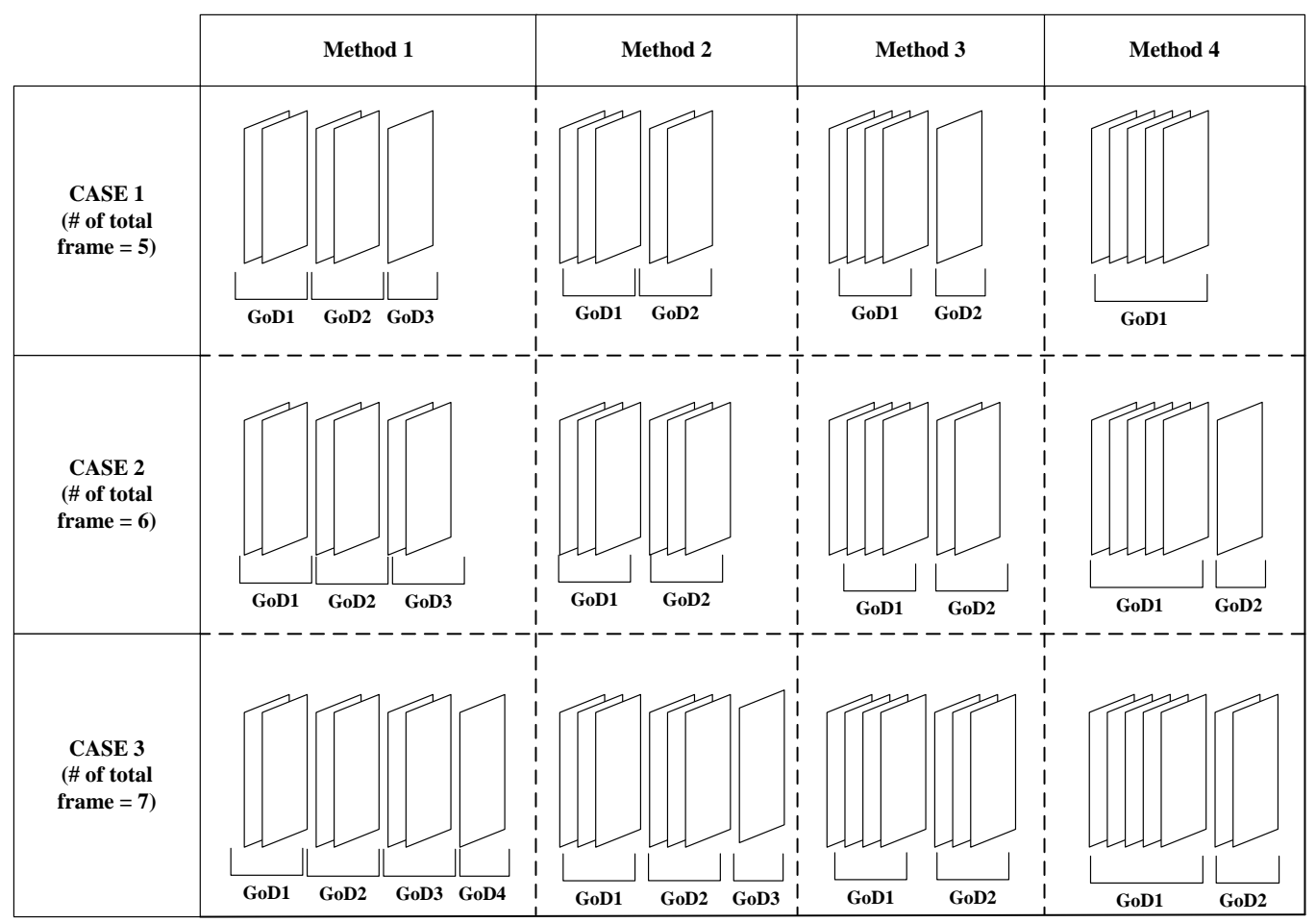

Figure 5. GoD Setting for DDTF CGH

(1) Method 1: The number of depth-map frames within the GoD was set as two, and the remaining depth-map frame was calculated using equation (1).

(2) Method 2: The number of depth-map frames within the GoD was set as three. If two depth-map frames were remaining, Method 1 was applied, and if only one frame was remaining, equation (1) was used for the calculation.

(3) Method 3: The number of depth-map frames within the GoD was set as four. If three depth-map frames were remaining, Method 2 was applied; if two were remaining, Method 1 was applied; and if one was remaining, equation (1) was used for the calculation.

(4) Method 4: The number of depth-map frames within the GoD was set as five. If four depth-map frames were remaining, Method 3 was applied; if three were remaining, Method 2 was applied; and if only one was remaining, equation (1) was used for the calculation.

After setting GoD's size, we performed the DDTF within the GoD. This procedure is shown in Figure 6. The DDTF estimates CC and DC by calculating the difference between the depth-map frames within the GoD (DG). Based on the generated coordinate information, in the case of CC, the CGH is calculated only once within one GoD, and in the case of DC, each instance is calculated. The reduction rate of the real amount of calculation differs by the amount of CC information in the GoD. Ultimately, the digital hologram is found by summing up the intermediate hologram values individually calculated according to the information of $\mathrm{CC}$ and DC. 


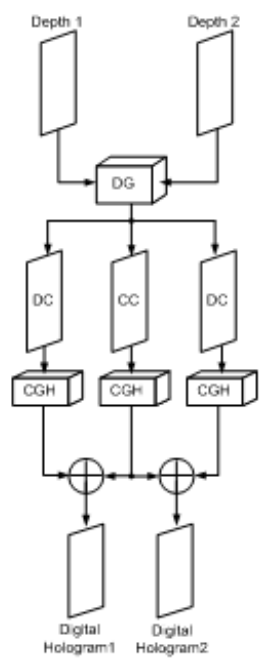

(a)

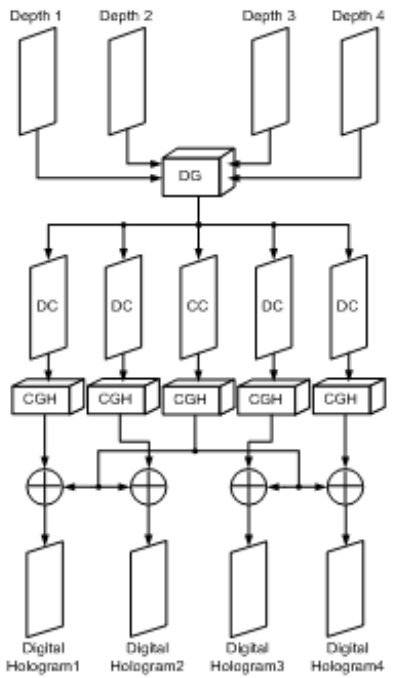

(c)

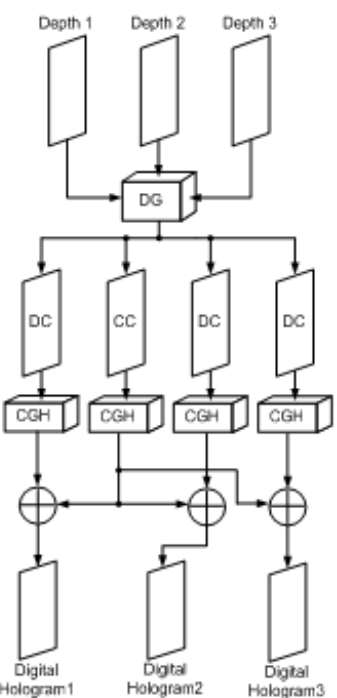

(b)
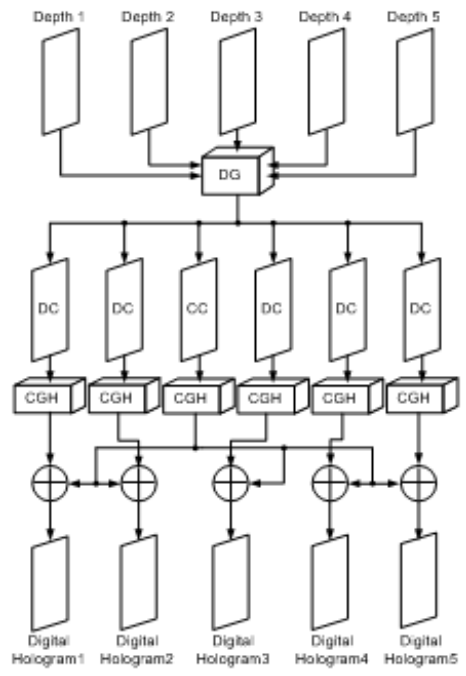

(d)

Figure 6. DDTF CGH: (a) Methods 1, (b) 2, (c) 3, and (d) 4

\section{Experimental Results}

The three depth-map videos shown in Figure 3 were used for verifying the performance of the proposed DDTF CGH algorithm in the GoD unit. Table 1 shows the experimental environment. Videos with different characteristics in resolution, frame rate, and moving direction of an object were used as the depth-map videos used in the experiment. CGH was performed after setting the GoD size to range from 2 to 5 frames to perform the DDTF. The experiments were performed for the depth-map videos of a rotating object in place and of a horizontally moving object, and the results were subsequently compared. 
Table 1. Experimental Environment

\begin{tabular}{|c|c|c|c|c|}
\hline Item & Sub-item & & Value & \\
\hline \multirow{5}{*}{ CGH parameter } & $\begin{array}{l}\text { Light sources \& depth frame } \\
{\left[\text { pixel }^{2}\right]}\end{array}$ & $\begin{array}{c}\text { Ballet } \\
200 \times 200\end{array}$ & $\begin{array}{c}\text { David } \\
176 \times 144 \\
\end{array}$ & $\begin{array}{c}\text { Camaro } \\
771 \times 826 \\
\end{array}$ \\
\hline & $\begin{array}{c}\text { Hologram resolution } \\
{\left[\text { pixel }^{2}\right]}\end{array}$ & & $1,024 \times 1,024$ & \\
\hline & Wavelength [nm] & & 633 & \\
\hline & $\begin{array}{l}\text { Pixel pitch } \\
{[\mu \mathrm{m}]}\end{array}$ & & 10.4 & \\
\hline & $\begin{array}{l}\text { Reconstruction distance } \\
{[\mathrm{cm}]}\end{array}$ & & 100 & \\
\hline \multirow{2}{*}{$\begin{array}{l}\text { Depth video } \\
\text { sequence }\end{array}$} & \# of frames & 11 & 360 & 38 \\
\hline & GoD size & & $2,3,4,5$ & \\
\hline
\end{tabular}

\subsection{Depth-map video of a rotating object}

The DDTF CGH algorithm was applied to the depth-map video in which an object rotating in place was recorded, as shown in Figures 3(a) and (b). To verify the performance of the proposed algorithm in various experimental environments, in the case of Figure 3(a), the temporal relationship between the adjacent frames was decreased using a low frame rate, and in the case of Figure 3(b), the temporal relationship between the adjacent frames was increased by setting a high frame rate.

Figure 7 shows the results of performing the DDTF CGH for each experimental data. To compare the performances, the required CPU time was measured after performing the CGH for each depth-map frame using equations (1) and (3), and the four methods of the DDTF $\mathrm{CGH}$ algorithm proposed in this paper were applied. The $\mathrm{x}$-domain of Figure 7 is a reconstruction based on the number of GoDs generated when Method 4 of the proposed algorithm is applied. For example, as shown in Figure 7(a), the number of GoDs is composed of three samples of $(4,4,3)$ when Method 4 is applied to Ballet's depth-map video composed of 11 frames. The CPU times were measured from these three samples for the other methods; similarly, the CPU times were measured from the three selected samples and were plotted. In Figure 7(b), the total frame number is 360 , and 72 GoDs are generated when Method 4 is applied. As shown in Figure 7, among the proposed methods, Method 1 showed the best result.

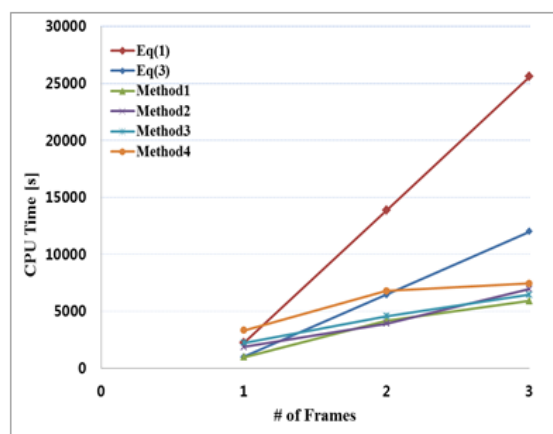

(a)

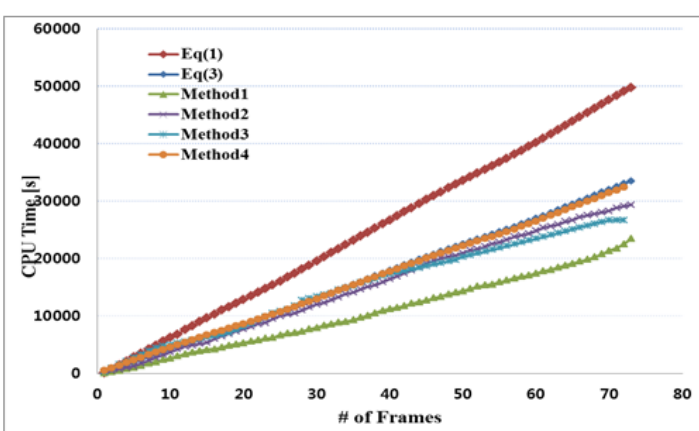

(b)

Figure 7. Results of Applying the Proposed Algorithm to the Depth-Map Video in which a Rotating Object is Recorded: (a) Ballet and (b) David 


\subsection{Depth-Map Video of a Horizontally Moving Object}

Figure 8 shows the result of comparing the proposed algorithm's performance for "Camaro" which is the depth-map video in which a horizontally moving object is recorded. In the case of the depth-map video in which a horizontally moving object was recorded, Method 1 showed the best result. In the case of Method 1, it was verified that the calculation speed was improved by approximately $69.22 \%$ as compared to equation (1) and by approximately $17.51 \%$ as compared to equation (2).

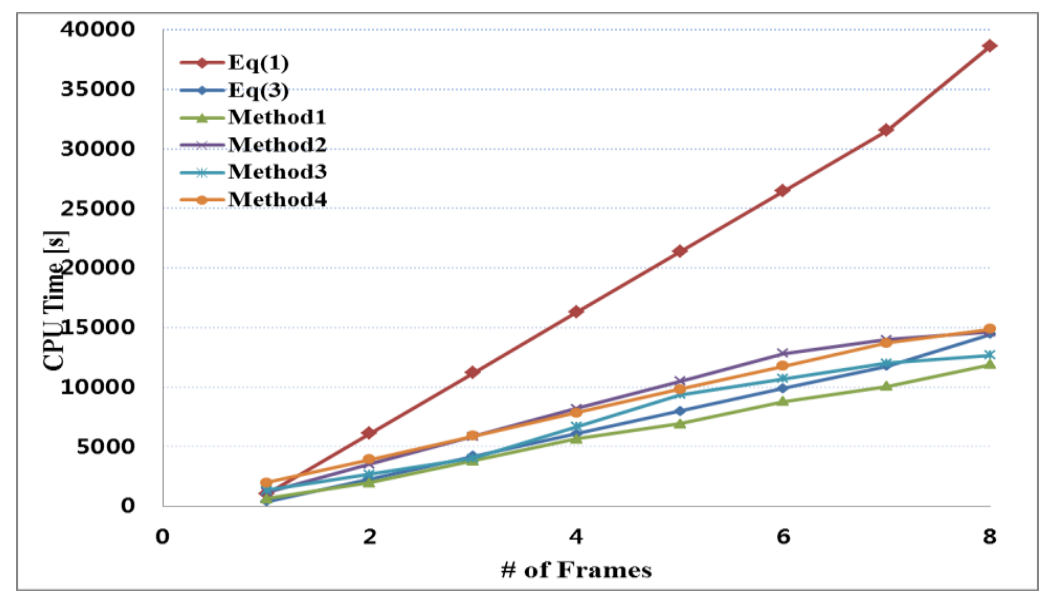

Figure 8. Results of Applying the Proposed Algorithm to the Depth-Map Video in which a Horizontally Moving Object is Recorded

\section{Conclusion}

In this paper, we proposed a method that could increase the CGH calculation speed, which is the most problematic issue in digital holographic video service models based on CGH. This method increased the CGH calculation speed by finding a temporal redundancy between the depth-map video frames through the DDTF process. Herein, the depth video frames were separated into GoD units, which were groups of two to four frames, and DDTF was performed for the frames in the same GoD.

The result of applying the proposed algorithm to three depth-map videos showed that the calculation speed increased by approximately $66 \%$ on average as compared to the results of independently performing $\mathrm{CGH}$ calculation on each depth video frame. In particular, in the case of the experimental result of Method 1 in which the GoD size was set to two, the calculation speed was increased by $76.89 \%$ in the case of Ballet, by $52.82 \%$ in the case of David, and by $69.22 \%$ in the case of Camaro.

We will continue this research to secure real-time capability of CGH calculation by applying the DDTF CGH algorithm in widely used processors such as graphic processor units (GPU) and field-programmable gate array (FPGA).

\section{Acknowledgements}

This work was supported by the National Research Foundation of Korea Grant funded by the Korean Government (MEST)" (NRF-2010-0026245) 


\section{References}

[1] H. S. Kim and I. G. Kim, "A Generation Method of MPEG-4 3D Scene for Mobile Environments", International Journal of Multimedia and Ubiquitous Engineering, vol. 6, no. 2, (2011), pp. 1-12, http://www.sersc.org/journals/IJMUE/vol6_no2_2011/1.pdf.

[2] W. Xu and E. J. Lee, "Continuous Gesture Recognition System Using Improved HMM Algorithm Based on 2D and 3D Space", International Journal of Multimedia and Ubiquitous Engineering, vol. 7, no. 2, (2012), pp. 335-340, http://www.sersc.org/journals/IJMUE/vol7_no2_2012/38.pdf.

[3] J. H. Shin and S. J. Lee, "An Analysis on the Degree of 3D Sense Following Distance and Location through 3D Depth Level Change", International Journal of Smart Home, vol. 6, no. 1, (2012), pp. 33 38, http://www.sersc.org/journals/IJSH/vol6_no2_2012/4.pdf.

[4] B. R. Brown and A. W. Lohmann, "Complex spatial filtering with binary masks", Appl. Opt., vol. 5, (1966), pp. 967-969, doi: http://dx.doi.org/10.1364/AO.5.000967.

[5] H. Yoshikawa, "Fast computation of Fresnel holograms employing difference", Opt. Rev., vol. 8, (2000), pp. 331-335, doi: http://dx.doi.org/10.1007/s10043-001-0331-y.

[6] T. Shimobaba and T. Ito, "An efficient computational method suitable for hardware of computer-generated hologram with phase computation by addition”, Comput. Phys. Commun., vol. 138, (2001), pp. 44-52, doi: http://dx.doi.org/10.1016/S0010-4655(01)00189-8.

[7] T. Shimobaba, T. Ito, N. Masuda, Y. Ichihashi and N. Takada, "Fast calculation of computergenerated-hologram on AMD HD5000 series GPU and OpenCL", Optics Express, vol. 18, no. 10, (2010), pp. 9955-9960, doi: http://dx.doi.org/10.1364/OE.18.009955.

[8] H. J. Choi, Y. H. Seo, S. W. Jang and D. W. Kim, "Analysis of Digital Hologram Rendering Using a Computational Method", Journal of information and communication convergence engineering, vol. 10, no. 2, (2012), pp. 205-209, doi: http://dx.doi.org/10.6109/jicce.2012.10.2.205.

[9] ISO/IEC 13818-2: 2000, "Information technology-generic coding of moving pictures and associated audio information-part 2: video", (2000).

\section{Author}

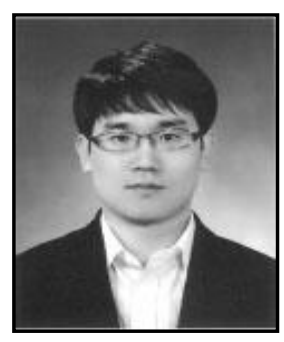

\section{Hyun-Jun Choi}

He received his M.S. and Ph.D. degrees in 2005 and 2009 from Dept. of Electronic Materials Engineering of Kwangwoon University in Seoul, Korea. He is currently an assistant professor with the department of Electronic Engineering, Mokpo National Maritime University. He is research interests are in optical image processing and $3 \mathrm{D}$ display. 